\title{
Cultural Diversity Dynamics
}

\author{
Elpida S. Tzafestas \\ Cognitive Science Laboratory \\ Department of Philosophy and History of Science \\ University of Athens \\ University Campus, Ano Ilisia 15771, Athens, GREECE \\ etzafestas@phs.uoa.gr
}

\begin{abstract}
In this work, we are exploring mechanisms that may contribute to cultural dynamics and cultural diversity. To this end, we are experimenting with an extended Axelrod model that uses a Moore neighborhood and heterogeneous sets of cultural features per agent. We are gradually introducing a number of psychologically realistic, basic and more advanced, conceptual models of cultural affinity perception and imitation and show that in many cases the population stabilizes to multi-cultural configurations. We also demonstrate the transitive advantage of two other mechanisms, attraction and spatial migration. In sum, our results show that a mix of initially differentially evolved or developed agents will not converge to monoculture, and that this is due to intrinsic psychological factors that are key players in behavioral change.
\end{abstract}

Keywords: Cultural simulation; Axelrod model; Selfishness; Indifference; Attraction; Migration; Transitive phenomena.

\section{INTRODUCTION}

Modern anthropology is concerned with understanding human culture and its many facets. Furthermore is it nowadays well-established that culture is not a monopoly of the human species, but other, supposedly lower, species engage in activities and show behaviors that depend on what may be called an animal culture in its own right [1]. Even more important, the modern behavioral synthesis assumes that evolution is not only biological, but cultural as well, and that the two evolutionary processes interact and show various synergies [2]. In this line of ideas, it is necessary to proceed to model complex biological-cultural systems, human or not. One important parameter in the whole continuum of target systems from low-level insect cultures to the most technologically advanced human ones, is diversity, which is all too often not well understood and taken for granted. A first research objective is to understand the mechanisms that generate and maintain cultural diversity and why and how they may have evolved. Why are human cultures so rich and diverse? Is this due to properties that are unique to human nature or are there pro-primate, even pro-social properties that are responsible for diversity generation and in what context or on what condition? A second objective is to understand how culture interacts with other social mechanisms or structures. The usual assumption that economic relations define or at least significantly influence cultural dynamics within a society, does not look sufficient anymore. On the contrary, it is obvious to us from first-person experience that economic rules, political structures, religious organization, language structures etc. are all factors that are both outcomes and agents of cultural and biological evolution. This appears to be parallel to what is observed in animal species, where the corresponding cultural features, such as tool-usage, nest site selection procedures, etc., are both outcomes and agents of biological evolution. Our short-term goal is therefore to delve into the mechanisms that generate, influence or maintain diversity. In this paper, we explore some related psychologically realistic issues that may be modeled in the context of a cultural simulation model.

Cultural simulation has been originally introduced by Axelrod [3] and subsequently developed and extended by several researchers, such as Shibanai, Trigg et al. $[4][5][6][7][8]$ According to this approach, an agent is characterized by a number of cultural features, each one of which may take one of several values, called traits. These features represent real-life properties such as if it is allowed to eat fish (boolean trait), favorite sport (many-valued trait), bow or shake to greet (boolean trait) etc. Various cultural systems may thus be defined as populations of agents sharing trait values with one another in all features, but with ample differences between groups. Initial modeling by Axelrod showed that an initially fully heterogeneous population (with random traits) may lead eventually to compact cultural groups that are homogeneous internally but with large differences between them, provided that a simple imitation process takes place: an agent may imitate (copy) a trait of a neighbor probabilistically. The larger the actual affinity between agents (coincidence of cultural traits), the larger the probability of another agent to interact with the agent. The original Axelrod model assumes a regular square two-dimensional grid where each position is occupied by a single agent and all interactions of an agent occur in a 4-neighbourhood: an agent selects randomly one of the four neighbouring agents (north, south, west or east of its position) to interact with. Interaction between two agents takes place with a probability that is equal to ( $\mathrm{F}$ $n) / F$, where $F$ is the number of cultural features and $n$ the number of features where the agents differ in trait. Interaction results in the initiating agent copying one of the different traits of its partner. This simple imitation process produces intricate diffusion dynamics that, surprisingly enough, do not lead to an homogeneous culture. Instead, stabilized spatial configurations in the grid are like the one given in fig. 1, where the color of the border between two grid places represents the degree of affinity between the two agents occupying these places: white 
color denotes $100 \%$ affinity (traits commonality), black color denotes 0 affinity and intermediate shades of gray denote intermediate degree of affinity (between 0 and 100\%). Equilibrium is reached when adjacent cultural groups have no traits in common and therefore interaction is not possible anymore. However, as has been put forward by other authors (for instance [9]) and we have verified experimentally ourselves, this result is a combined side-effect of the model assumptions of 4-connectivity and fewer features than traits per feature. If instead, more features are used with fewer traits each, all systems eventually lead to monoculture. On top of this, if 8-connectivity (Moore neighborhood) is assumed, systems converge to full affinity substantially faster. It is this modeling intricacy that led us to reflect initially on the factors that may be responsible for the emergence of diverse cultural groups.

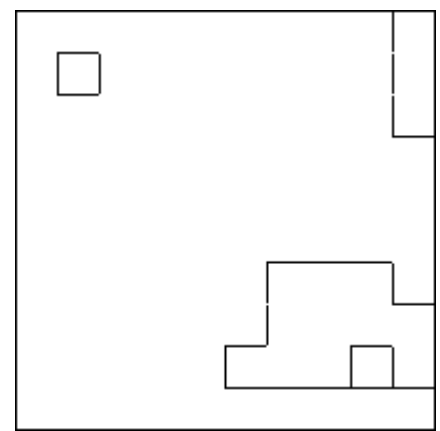

Figure 1. Typical stable outcome of the original Axelrod model in a 10x10 world with 5 cultural features of 10 traits each (after 150000 cycles).

Section II presents a modified Axelrod model and various personalized models that are initially thought to promote diversity, while section III studies the effects a series of advanced psychological models that are purported to make the socio-cultural model more realistic. Section IV presents some additional mechanisms that may be involved in diversity generation and persistence, and finally section $\mathrm{V}$ concludes by discussing the results and drawing some directions for future research.

\section{MODIFIED AXELROD MODEL}

\section{A. Diversified Number of Features and Traits}

In this basic Axelrod model, all agents have the same features with diverse initial traits for each feature. However, this is only a little realistic; in practice, people in a multicultural context may also have different features, beside different traits. For example, in some non-western cultures, hats or jewels may be of different kinds, denoting various functions, or even societal ranks, while in western cultures there is no such notion and these features will go unnoticed. We expect agents in a mixed context to perceive and develop affinity (common traits) with "kin" in terms of common features and remain heterogeneous as far as uncommon features are concerned. In this case, cultural diversity will be persistent and will be due to heterogeneity in cultural features. We have modeled this option as agents having an array of $\mathrm{M}$ features, out of which only the $\mathrm{K}$ first features are active. We have run experiments with $\mathrm{M}=15$ and $\mathrm{K}$ heterogeneous (up to $\mathrm{M})$. Affinity between two agents is computed on the maximum number of features of both agents $\left(n / \max \left(F_{1}, F_{2}\right)\right.$, where $F_{i}$ is the number of features of agent-i and $n$ the number of features where the agents differ in trait value) but imitation takes place only on the $\min \left(\mathrm{F}_{1}, \mathrm{~F}_{2}\right)$ common features. The results are presented in fig. 2 and show that heterogeneous populations stabilize in medium affinities between agents, unlike homogenous populations that converge to full affinity. Final configurations in heterogeneous cases are also much more diverse apparently than final configurations in the original Axelrod model of homogeneous populations where interactions assume 4-connectivity (compare fig. 1 with fig. 2). This model therefore shows that cultural diversity may be due to the presence of uncommon features rather than to brittle spatial interaction modeling and specific initial conditions.
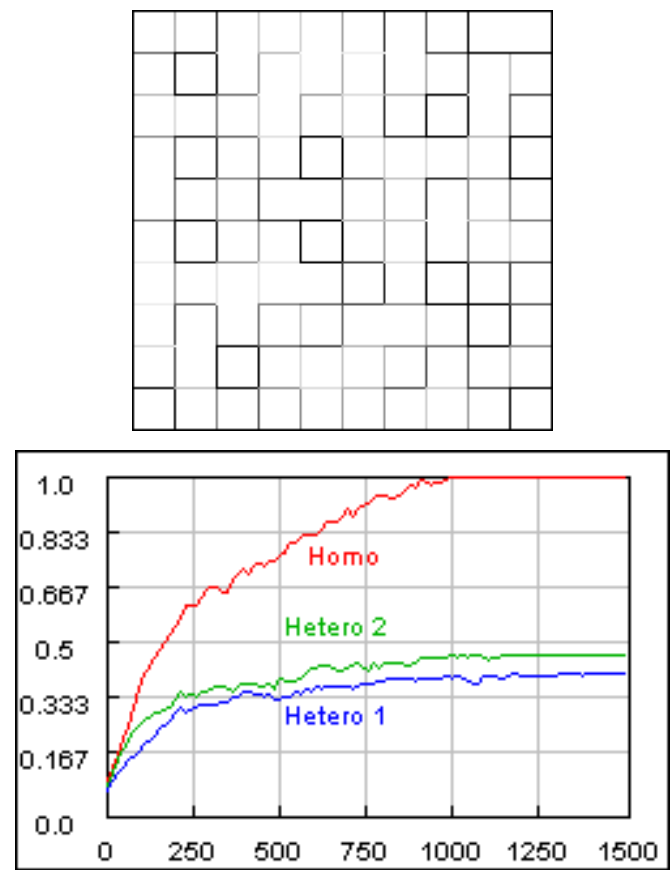

Figure 2. (x: time in 100s of cycles, y: affinities) Typical outcomes of the modified Axelrod model in a 10x10 world with Moore neighborhood and up to 15 cultural features of up to 10 traits each (after 150000 cycles). The homogeneous case (all agents with exactly 15 features of 10 exactly traits each) leads to full affinity. Heterogeneous cases (features with different numbers of traits, and different number of features per agent) stabilize to partial affinity.

\section{B. Personalized Models}

The previous heterogeneous model is inaccurate in using an "ordered" set of features, where agents whose number of features differs by one are forced to have only one uncommon feature. For example, if agent- 1 has 5 features and agent- 2 has 6 features, then these features are respectively $\{1,2,3,4,5\}$ and $\{1,2,3,4,5,6\}$, and not for instance $\{1,3,5,6,8\}$ and $\{2,3,4,7,9,10\}$. Therefore, it is straightforward to think about relaxing this model and allowing free initialization of features in individual agents. Then, this arrangement raises the question of how individual agents will tackle uncommon features and how they will compute affinity. 
The obvious option is to have agents compute affinity on common features and define 0 affinity for uncommon features. Thus for two agents with features $\left[\mathrm{T}_{1}, \mathrm{~T}_{2},--,--, \mathrm{T}_{5},--, \mathrm{T}_{7},--, \mathrm{T}_{9}, \mathrm{~T}_{10}\right]$ and $\left[--, \mathrm{T}_{2}, \mathrm{~T}_{3},--, \mathrm{T}_{5},--, \mathrm{T}_{7}, \mathrm{~T}_{8},--, \mathrm{T}_{10}\right]$, both agents will compute partial affinities as [0,aff,0,--,aff,--,aff, 0,0, aff $]$, where aff denotes the regular Axelrod affinity for the corresponding trait. This leads to very quick stabilization to very low affinities for all agents, because most of the active features of an agent won't be shared with others. The psychologically valid affinity computation method is therefore to define the above partial affinities vector as [--,aff,--,--,aff,--,aff,--,--,aff], where affinity is computed only on common features and any feature perceived as not shared by an agent does not count. This selfish model yields the results of fig. 3, where heterogeneous populations converge much faster to full affinity, because affinity is computed only on shared features that converge therefore very fast. Results are taken with a modified Axelrod model in a 10x10 world with Moore neighborhood and up to 15 features of up to 10 traits each. Unless otherwise stated, all experiments reported use the same parameters.

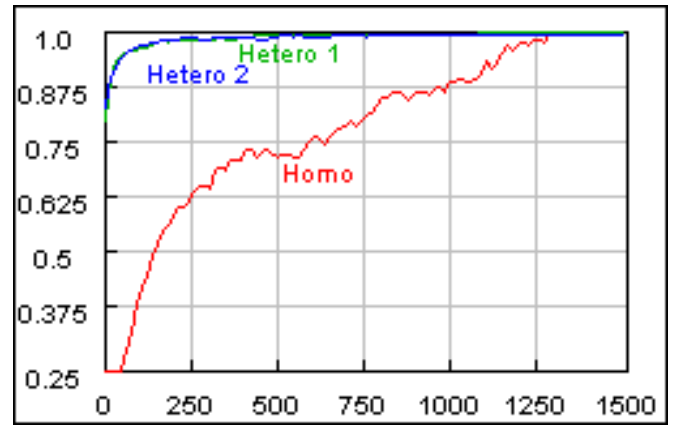

Figure 3. (x: time in 100s of cycles, y: affinities) Typical outcome for a modified Axelrod model with selfish agents. The heterogeneous cases converge to full affinity nuch faster than the homogeneous case.

Still, this model is homogeneous in the treatment of uncommon features, in the sense that a feature that is active for one agent and inactive for a second does not participate in affinity computation for either one of the agents. It seems more psychologically realistic to assume that agents compute affinities with neighbours in an individualistic manner that differs from agent to agent: an agent A will compute affinity with agent B only on A's features (and affinity will be 0 if A does not possess the corresponding feature), while $B$ will compute affinity with agent A only on B's features. In the previous example, the partial affinity computation vector for the two agents will become respectively [0,aff,--,--,aff,--,aff,-, 0, aff] and [--,aff, $0,--$, aff,--, aff, $0,--$, aff]. This second selfish model does not differ from the previous one in homogeneous populations. In heterogeneous populations, however, it shows a complex behavior, because the actual affinity (as perceived by an external observer), that is computed as before, will converge fast to full affinity, so that common traits will coincide, whereas the perceived affinity by each agent will be different and markedly lower. Fig. 4 shows how global affinity converges to an intermediate value and how neighboring agents may have different views of their affinity with one another.

A further development of psychologically realistic models is to assume that agents will handle differently the various cultural features, namely that there will be important features that contribute to their perception of affinity and others that do not count. The observation behind this development is that in some cultures people are indifferent to a particular trait, for example in modern western cultures, people are generally indifferent to whether a woman wears a skirt, a dress or trousers, while in other cultures this is not the case. Translated to everyday practice, this means that people do not regard themselves as different from others that have any trait value for this feature, and actually on many occasions people will temporarily align with their neighbors' behavior, precisely because they are indifferent to the particular trait values.
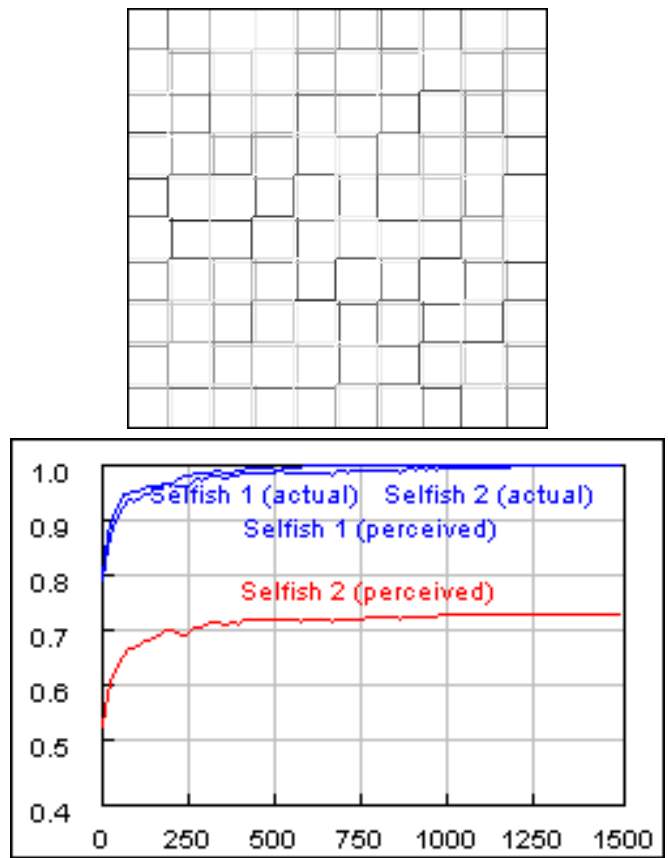

Figure 4. Comparison of the first and second selfish model. (above) Perceived affinities in the final configuration for the second selfish model: every two neighboring agents regard their own relation and affinity differently - two lines are shown in each border instead of one, each line showing the perceived affinity by the closest agent (below, x: time in 100s of cycles, y: affinities) In the first selfish model, actual and perceived affinity are the same and converge to full affinity. In the second selfish model, perceived affinity is much lower than the actual affinity that converges to full affinity.

This indifferent model is implemented via the use of an additional dont-care vector storing a boolean value foreach feature (true for indifference). For instance, for two agents with 10 features (named $\mathrm{T}_{1}$ to $\mathrm{T}_{10}$ ) and dont-care vectors respectively $[\mathrm{t}, \mathrm{f}, \mathrm{f}, \mathrm{t}, \mathrm{t}, \mathrm{f}, \mathrm{f}, \mathrm{t}, \mathrm{t}, \mathrm{t}] \quad$ and $[\mathrm{f}, \mathrm{t}, \mathrm{f}, \mathrm{t}, \mathrm{t}, \mathrm{t}, \mathrm{f}, \mathrm{f}, \mathrm{f}, \mathrm{t}]$, the corresponding partial affinity vector will be computed as [aff,aff,aff,--,--, aff,aff,aff,aff,--]. Again, this supposes an homogeneous feature handling method, whereas features for which both agents are indifferent do not count. As before, we define a second indifferent model, where the dont-care values are treated in an individualistic manner; in the previous example, the partial affinity vectors of the two agents become [--,aff,aff,--,--, aff,aff,--,--,--] and [aff,--,aff,--,--,--,aff,aff,aff,-], respectively. Results are given in fig. 5 and fig. 6, respectively. Again, the homogeneous population does not differ from previous cases, while heterogeneous ones present the inverse behavioral pattern as the one found in selfish models: in indifferent models, actual affinities are low, because of the different active features and dont-care values per agent, 
whereas perceived affinities are high, because only features shared in terms of dont-care value take part in affinity computation. Finally, fig. 7 gives the results of mixed population, consisting of both selfish and indifferent agents. Notably in this case, the average perceived affinity is substantially higher than the average actual affinity, which means that there is cultural diversity that is not perceived as harmful (perceived affinity is high). This is sofar the most psychologically realistic model and its results are the closest to real-life observations, although not in the sense that the results are validated with real human-level data, but rather in the sense that conceptually/functionally they reflect our common understanding of cultural tolerance and indifference, better than the previous models. The realism stems principally from the fact that it is in practice impossible for an agent to know another agent's dont-care value, i.e., to know how it reasons internally. Because such knowledge is approximate, if any, it makes sense to compute affinity and act individually based mainly on own terms and perceptions.

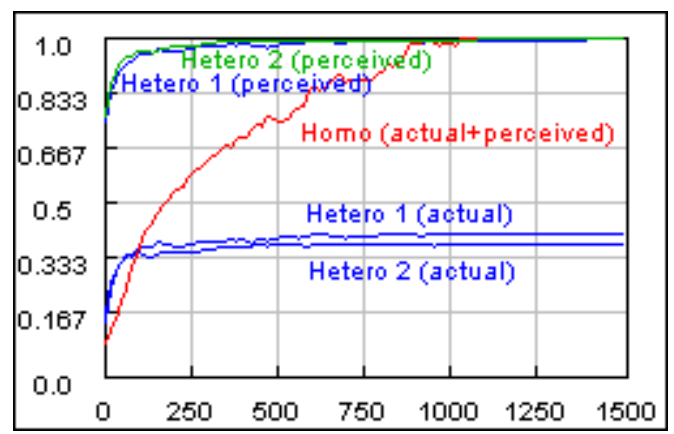

Figure 5. (x: time in 100s of cycles, y: affinities) Typical outcome for a modified Axelrod model with indifferent agents. The heterogeneous cases converge much faster than the homogeneous case, but they converge to full perceived and partial actual affinity.

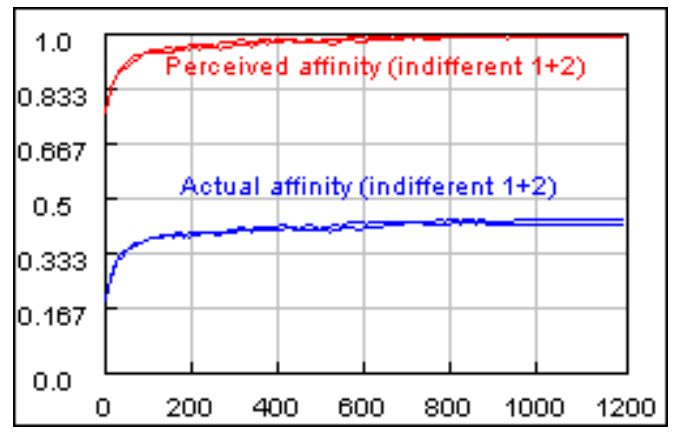

Figure 6. (x: time in 100s of cycles, y: affinities) Comparison of the first and second indifferent model. Both converge to full perceived affinity but partial actual affinity.

\section{ADVANCED PSYCHOLOGICAL MODELS}

Despite their conceptual realism, the previous models are insufficient for modeling cultural phenomena and diversity. In reality, we expect agents to reason on a continuum between care/dont-care behavior. Furthermore, we expect agents to adapt individually to other agents' cultural preferences by modifying their own care/dont-care relation.

\section{A. Complex Model}

We may define a new model that uses degrees of indifference toward cultural features. Each cultural feature is assigned a real-valued weight between 0 and 1: the lower its value, the more indifference the agent will show toward the feature. The perceived affinity of an agent with another one is defined accordingly as $\Sigma \mathrm{w}_{\mathrm{i}} a f f_{\mathrm{i}} / \Sigma \mathrm{w}_{\mathrm{i}}$, while the actual affinity is defined as usually $((F-n) / F)$. Apparently, with this model it is very common to have agents that perceive each other very differently due to their different weight vectors. Typical results of five independent runs are given in fig. 8. Average actual affinity rises to full affinity (common features become uniform across the population), while average perceived affinity stabilizes to far lower values (not common features have different trait value for different agents). As before, the presence of features that are not shared across the population, creates diversity that is furthermore perceived differently by individual agents.

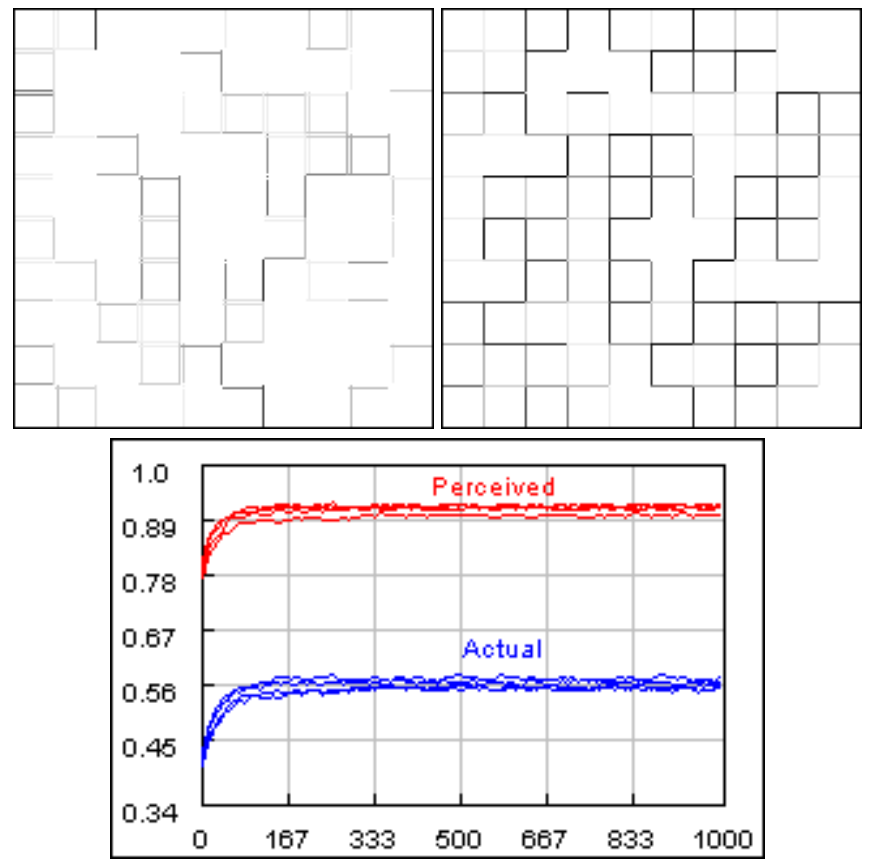

Figure 7. (x: time in 100s of cycles, $\mathrm{y}$ : affinities) (above left) Final perceived affinities. (above right) Final actual affinities. (below) Typical outcome for a modified Axelrod model with a mix of selfish and indifferent agents (5 runs). All converge to high perceived affinity but significantly lower actual affinity.

\section{B. Meta-Models}

Because all models are using a vector indicating how an agent perceives the importance of various cultural features, it would be natural to ask whether the vectors themselves may be regarded as cultural features, prone to be imitated. This observation stems from our everyday experience with cultural change which is often enough change in habits about thinking about culture. It makes sense to assume that an agent may safely change by copying a cultural feature of no true importance to him. This way, an agent may hope to achieve better affinity with another agent at no personal expense. In our models, this is implemented as imitation of a feature importance parameter for a cultural feature that is at first not important for the agent. In the case of the selfish models, this 
amounts to imitation of the feature for which the agent has a NONE value (what is depicted as '--' in the feature vector). In the case of the indifferent models, it is imitation of another agent's dont-care value for a feature for which dont-care=true (in the case of the indifferent models). Finally, in the case of the complex model, it is imitation of another agent's different weight.
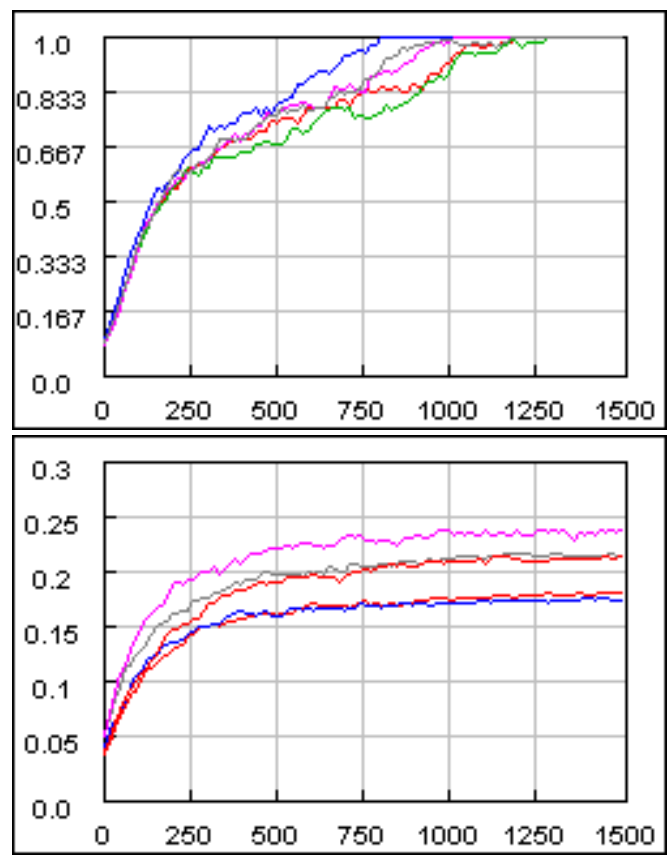

Figure 8. (x: time in 100s of cycles, y: affinities) Typical outcome for a modified Axelrod model with complex agents (5 runs). (above) Average actual affinity converges to full affinity. (below) Average perceived affinity stabilizes to intermediate values.

Figs. 9 to 11 present typical results with meta-selfish, metaindifferent or meta-complex models. In all experiments, in each interaction there is a $50 \%$ probability to imitate a shared feature and a $50 \%$ probability to meta-imitate, i.e. to imitate the importance value for an uninteresting feature. Interestingly, contrary to common belief, the meta mechanism as implemented has not much to offer. In the selfish case, the average affinity converges always to full affinity but the speed of convergence does not change. In the indifferent case where there is large perceived affinity that does not follow actual realworld affinity as observed from the outside, the average actual affinity does not rise with meta-behavior. Finally, in the complex case, average affinity slightly worsens. Therefore, in all cases, copying traits that are otherwise indifferent for the agent induces neither better perceived affinity nor better actual affinity and cultural diversity remains intact or is even amplified. This is exemplified below where initial and final trait and dont-care vectors for two agents are given. Notice that the number of dont-cares rises, while the rest of the traits are shared.

\section{At start \\ Agent 1

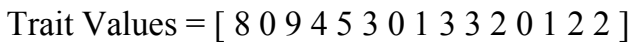 \\ Dont Care $=[$ F F F F F F T F F F F F F F F $]$}

Agent 2

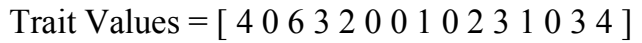

Dont Care $=[$ F T T F F T T F F T F T F F F $]$

\section{At end}

Agent 1

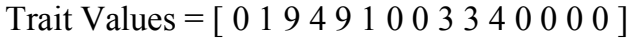

Dont Care $=[$ T F T T T F T F F F F F F F F ]

Agent 2

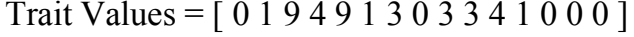

Dont Care $=[$ T F T T T F T F F F F T F F F $]$

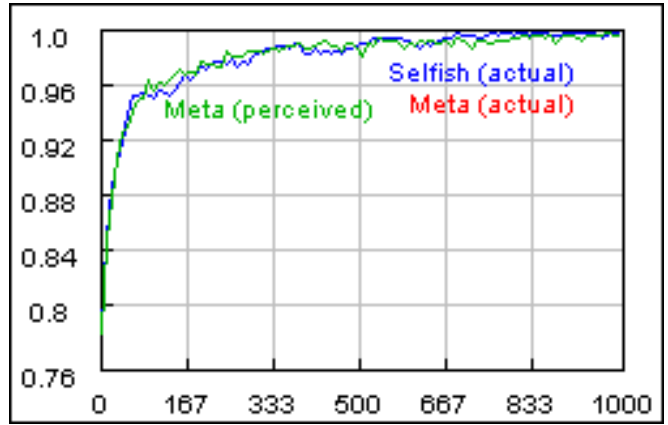

Figure 9. (x: time in 100s of cycles, y: affinities) Typical outcome for a modified Axelrod model with selfish agents (with and without meta). The results with and without meta do not differ.

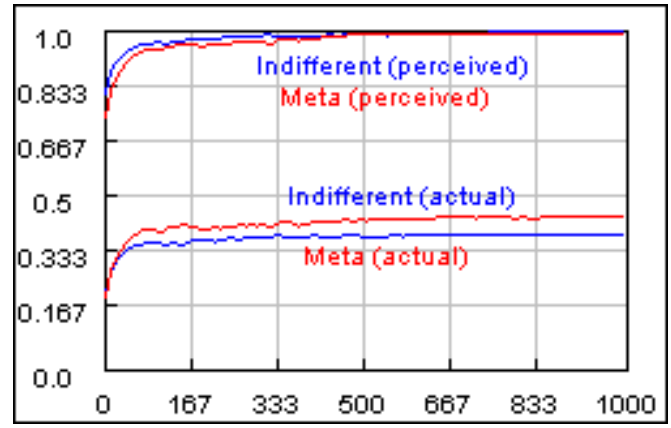

Figure 10. (x: time in 100s of cycles, y: affinities) Typical outcome for a modified Axelrod model with indifferent agents (with and without meta). Final qualitative results do not differ, but meta yields slightly lower perceived affinity and slightly higher actual affinity.

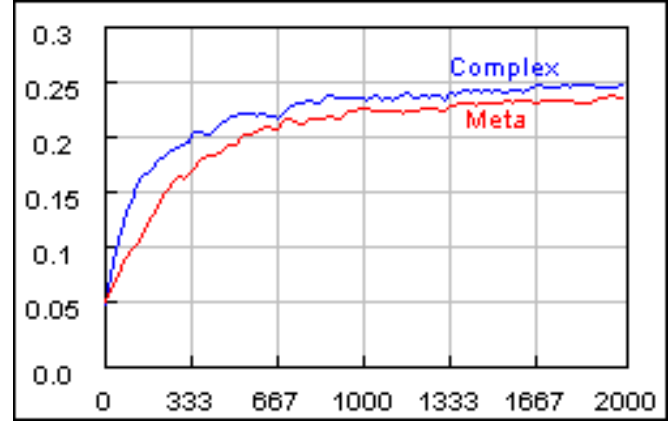

Figure 11. (x: time in 100s of cycles, y: affinities) Typical outcome for a modified Axelrod model with complex agents (with and without meta). Actual affinity is slightly lower in the meta case.

\section{Blind Adaptation}

Apart from directed change in the sense introduced above, there are also reflexive or blind psychological mechanisms 
leading to behavioral change. One such mechanism is a psychological defense mechanism that induces changes that make an agent increasingly tolerant to difference, i.e. increasingly indifferent. In the case of the three models, selfish, indifferent and complex, this translates respectively to setting a trait to NONE, setting a dont-care value to true and reducing a weight (for example, by half). This blind adaptation mechanism is expected to lead to higher affinity with other agents. Figs. 12 to 14 present typical results with adaptiveselfish, adaptive-selfish 2 and adaptive-indifferent models. In all cases, adaptation speeds up stabilization significantly. The final outcome (average affinity) with adaptation differs from that without adaptation in the case of the models not reaching full actual or perceived affinity. For the selfish 2 model, average perceived affinity improves with adaptation, whereas for the indifferent model, the average actual affinity becomes worse. This is exemplified below where initial and final feature and dont-care vectors for two agents are given. Notice how dramatically the number of dont-cares has risen, while the rest of the traits are shared. Thus, (actual) cultural diversity is amplified by mechanisms, such as a blind psychological defense mechanism, whereby agents devise ways to be individually happy (perceive full affinity with neighbors) despite actual, externally observable, discrepancies.
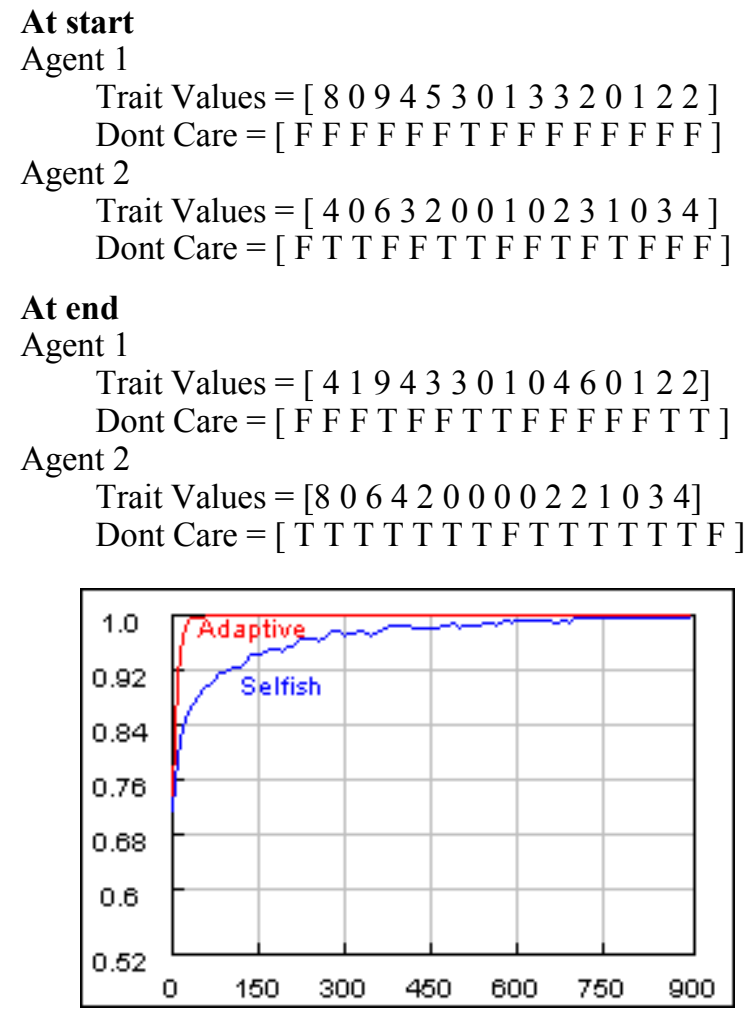

Figure 12. (x: time in 100s of cycles, y: affinities) Typical outcome for a modified Axelrod model with selfish agents (with and without adaptation). The adaptive model converges extremely fast to full actual affinity.

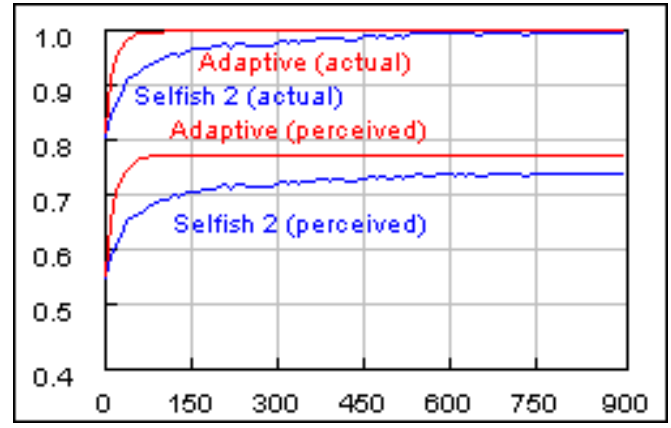

Figure 13. (x: time in 100s of cycles, y: affinities) Typical outcome for a modified Axelrod model with selfish 2 agents (with and without adaptation). Final qualitative results do not differ, but the adaptive model is extremely fast and yields higher affinities, both perceived and actual.

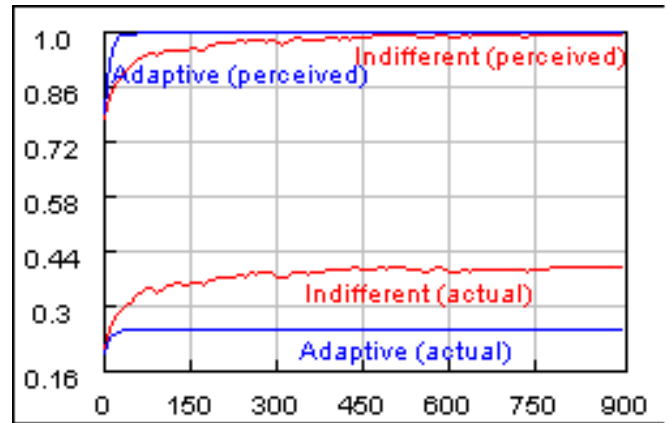

Figure 14. (x: time in 100s of cycles, y: affinities) Typical outcome for a modified Axelrod model with indifferent agents (with and without adaptation). Final qualitative results do not differ, but the adaptive model yields markedly lower actual affinity and slightly higher perceived affinity.

\section{ADDITIONAL MECHANISMS}

In the past we have experimented with an attraction model [10] shown to compensate for initial behavioral differences. This attraction model relies on a network of attraction relations, where each agent is attracted by a number of other agents. The overall result of attraction for an agent will be to unconditionally cooperate with another agent or imitate it or perform any other action that normally necessitates some reasoning in order to be launched. It may be conjectured that this completely reactive behavior may have evolved as a functional, fast, low-cost behavior that shortcuts expensive reasoning [11]. In the case of cultural imitation, attraction will show as unconditional imitation of another agent, irrespective of perceived affinity. Once more, this is supported by real-life observations, where people are easily adopting theoretically remote cultural traits because of attachment to other people. Counter-intuitively, in our experiments, attraction contributes only a little to actual affinity in models where actual and perceived affinities differ. In the case of the complex model (results are depicted in fig. 15), attraction yields minor benefits compared to the basic model, except in terms of convergence speed. Note that in this case, the blind adaptation mechanism of the previous section is worse than the basic complex model both in terms of final affinity level reached and in terms of speed. Thus the attraction mechanism as implemented is not actually a contributor to cultural globalization at a population level, despite the expectation from the popular view. 
Our final concern is spatial mobility. So far, all agents are supposed to be sedentary and have a fixed position on the grid that defines their interactions with neighbors. However, it is natural to introduce at least some limited mobility to the agents behavior. We have implemented mobility in our model as exchange of positions of two random agents in the grid with a small probability. This migration mechanism has a dramatic influence on the convergence speed and on the shape of the transitive behavior, but no visible influence on the final levels that actual or perceived affinities reach, as fig. 16 shows. Obviously, such behavioral "diffusion" inside the grid converts the original grid spatial configuration to a social networking one with the potential to show some of the interesting results reported in the related literature [12], but outside the scope of behavioral modeling of cultural imitation as such.

From the above, it follows that the attraction and migration mechanisms are orthogonal to one another and that they influence the transitive behavior of a population of agents, rather than the final levels reached. At first this looks as an indifferent result. However, this is not true, because real life appears like a transitive stage anyway and all observed behavior is produced in systems that are not in a stable situation and can never be, since the physical or cultural environment changes constantly.

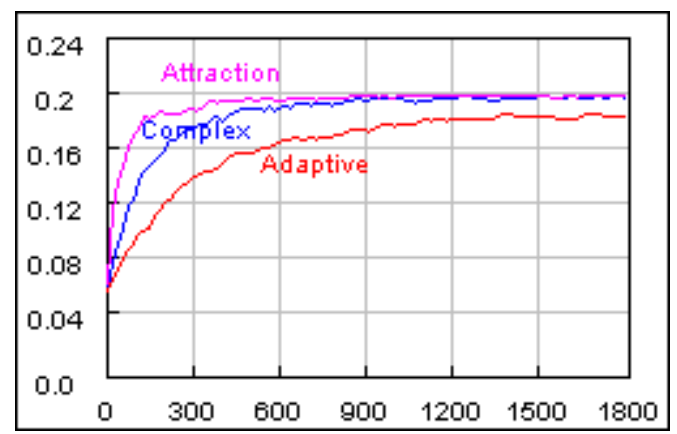

Figure 15. (x: time in 100s of cycles, y: affinities) Comparison of the effects of adaptation and attraction on a complex model. The final qualitative result is the same, but attraction yields slightly higher actual affinity, while adaptation yields clearly lower actual affinity.

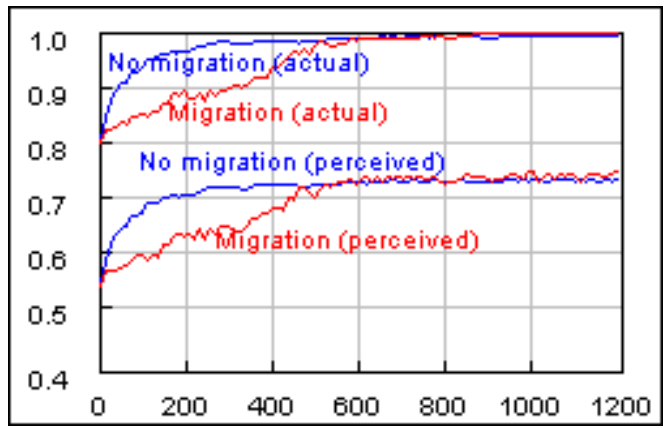

Figure 16. (x: time in 100s of cycles, y: affinities) The effect of migration on a selfish 2 model. The qualitative results with and without migration do not differ, however migration is responsible for a long transition period before stabilization and for much lower affinities (both actual and partial) within that period.

\section{DISCUSSION}

In this paper we have studied a modified Axelrod model of cultural simulation, where the agents have heterogeneous cultural features and these features have different structure (different number of traits per feature). This configuration corresponds to populations where many agents of different social and evolutionary origins have to co-exist, as is for example the case of places that have received many different immigrant populations. In this case, and unlike what may be commonly thought, initial diversity does not disappear and the population does neither become monocultural, nor does it stabilize to well-delimited cultural groups; rather cultural affinity between adjacent agents is large enough but not perfect. This is not only due to the initial diversity but mainly to the individualistic behavior of the agents that perceive each other differently (these individual differences may be attributed to personality or cultural differences). Furthermore, even if we assume that the perception of the other is a cultural feature per se (actually a meta-feature) that may be also object of cultural imitation, the dynamics don't change significantly. Psychological defense mechanisms of adaptation only worsen the situation, and the population reaches lower levels of actual affinity, as observed by an external observer. Finally, reactive pro-social mechanisms such as attraction and migratory behavior delay the transitive behavior of the population, which is an interesting result, because in reality cultural features are not fixed for a long period. Technological innovation, inventions, artistic creations, social/economic/political opportunities of all kinds etc. are factors that contribute to a constantly changing landscape of cultural features, so that a population is in a transitive state forever. In this transitive state, it is therefore shown that mechanisms such as attraction and processes such as migration pull the dynamics of cultural change in different directions. Overall, our experiments show that cultural diversity is maintained by individualistic behaviors at a meta level, that act not on cultural content (traits) but on cultural structure (features), and that these behaviors correspond to psychological reflexes or other impulsive innate mechanisms. The main innovation in comparison with other cultural models lies in the presence of individually perceived affinities that do not necessarily coincide with one another or with an externally observable view. Therefore an intrinsically stable environment may be perceived from the outside as a rich, potentially explosive environment. For this reason, such an environment shows a high degree of autonomy from a systemic point of view and cannot be easily manipulated from the outside, because the underlying causal factors are not exposed to the outside.

As a first set of subsequent experiments, we may envisage extensions such as more precise models of meta-imitation and adaptation, or cultural features that control how attraction influences behavior, or migratory agents whose openness to imitation is variable and may be also a cultural feature, etc. At a next stage, we would like to experiment with meta-cultures, i.e. culturally encoded rules of cultural perception and change, that are a result of long-term development within a cultural context, cannot be directly observed and thus cannot be imitated. Practical constraints of cultural imitation are also interesting to explore, such as exposure to common 
environmental or social factors, at first glance unrelated to culture.

This future work aims at gaining more understanding of the factors and processes that generate, influence and maintain cultural diversity within a population and of cultural diversity dynamics.

\section{REFERENCES}

[1] K.N. Laland, V.M. Janik, "The animal cultures debate", Trends in Ecology and Evolution, 21(10), pp. 542-547, 2006.

[2] J. Gintis, "A framework for the unification of the behavioral sciences", Behavioral and Brain Sciences, 30(10), 2007, pp. 1-61.

[3] R. Axelrod, "The dissemination of culture: A model with local convergence and global polarization", The Journal of Conflict Resolution, 41(2):203-226, 1997.

[4] Y. Shibanai, S. Yasuno, I. Ishiguro, "Effects of global information feedback on diversity: Extensions to Axelrod's adaptive culture model", The Journal of Conflict Resolution, 45(1), 2001, pp. 80-96.

[5] M. San Miguel, V.M. Eguiluz, R. Toral, K. Klemm, "Binary and multivariate stochastic models pf consensus formation", IEEE Computing in Science and Engineering, Nov./Dec. 2005, pp. 20-26.
[6] S. Vitzthum, "Dissemination of culture in a networked world: The influence of network structure on the outcomes of the Axelrod cultural model", Proceedings Americaas Conference on Informarion Systems (AMCIS), 2007.

[7] A.B. Trigg, A.J. Bertie, S.F. Himmelweit, "Modelling Bourdieu: An extension of the Axelrod cultural diffusion model", CRESC, Open University, Working Paper No. 49, 2008.

[8] L.R. Peres, J.F. Fontanari, "The mass media destabilizes the cultural homogenous regime in Axelrod's model", Journal of Physics A; Mathematical and Theoretical, 43(2010).

[9] N. Lanchier, "The Axelrod model for the dissemination of culture revisited", arXiv:1004.0365v1, 2 Apr.2010.

[10] E.S. Tzafestas, "Attraction and cooperation in noisy environments", Proceedings 6th International Conference on Computational Intelligence in Economics and Finance (CIEF 2007), Joint Conference on Information Sciences, June 2007, Salt Lake, Utah, USA.

[11] E.S. Tzafestas, "Selection for attraction", Proceedings of the Workshop "Extending the Darwinian Framework: New levels of selection and inheritance", European Conference on Artificial Life 2007, September 2007, Lisbon.

[12] N. Ganguly, A. Deutsch, A. Mukherjee, Dynamics on and of complex networks (Applications to biology, computer science and the social sciences). Boston, Birkhäuser, 2009. 